Hong and colleagues synthesized the polymer by atom transfer radical polymerization. They used a modified pyrene as the initiator, which fixes the fluorophore onto one end of the growing chain, and chose sulfadimethoxine as their monomer for its physiological-range dissociation constant. After polymerization, coumarin 343 was added to the free end of the chain through an ester linkage. At pH 7.5 and above, the primary emission is from the pyrene, as would be expected from the excitation wavelength of $334 \mathrm{~nm}$; at pH 7.0 and below, the researchers observed a broad peak at $491 \mathrm{~nm}$, corresponding to coumarin 343 emission as well. Since coumarin 343 is not excited by 334-nm light, the researchers ascribe this peak to a fluorescence resonance energy transfer from the pyrene to the coumarin due to physical proximity. This occurs as the $\mathrm{pH}$ is lowered below the dissociation constant of the polymer chain and the chain transitions from a coil to a globule state, pulling the two fluorophores close together. According to the researchers, this process and the appearance of the signal peak from the coumarin are reversible, suggesting that this sensor could be used to monitor multiple $\mathrm{pH}$ changes over time.

KRISTA L. NIECE

\section{New Family of Self-Assembled Nanolattices Created}

Inspired by the way most solids form in nature, with free-floating molecules spontaneously assembling themselves into a rigid, highly uniform array, researchers from Columbia University and IBM have learned how to create a new family of intricate structures out of artificial nanoscale crystals. Because the nanocrystals can be chosen for their precise magnetic and electronic properties, these new structures could have broad application for magnetic storage and nanoscale electronics.

"You can think of nanocrystals as building blocks like the toy Lego," said the group's spokesperson, Stephen O'Brien of Columbia, "in which a larger structure can be assembled by locking in the pieces according to their shape and the way they prefer to join to each other. Except all of this is on an incredibly small length scale-billionths of a meter."

The Columbia/IBM team has borrowed ideas from the natural world, in which the right conditions can cause the slow growth of highly uniform structures built from miniature building blocks. Opals are an example of this phenomenon: opals consist of tiny spherical building blocks of silica packed into an ordered structure.
As reported in the January 5 issue of Nature (DOI: 10.1038/nature04414; p. 55), $\mathrm{O}^{\prime}$ Brien and postdoctoral research scientist Elena Shevchenko of Columbia; researchers Dmitri Talapin and Christopher Murray at the IBM Watson Research Center in Yorktown Heights, New York; and Nicholas Kotov of the University of Michigan, Ann Arbor, have formed more than 15 different binary nanoparticle superlattice (BNSL) structures using combinations of semiconducting, metallic, and
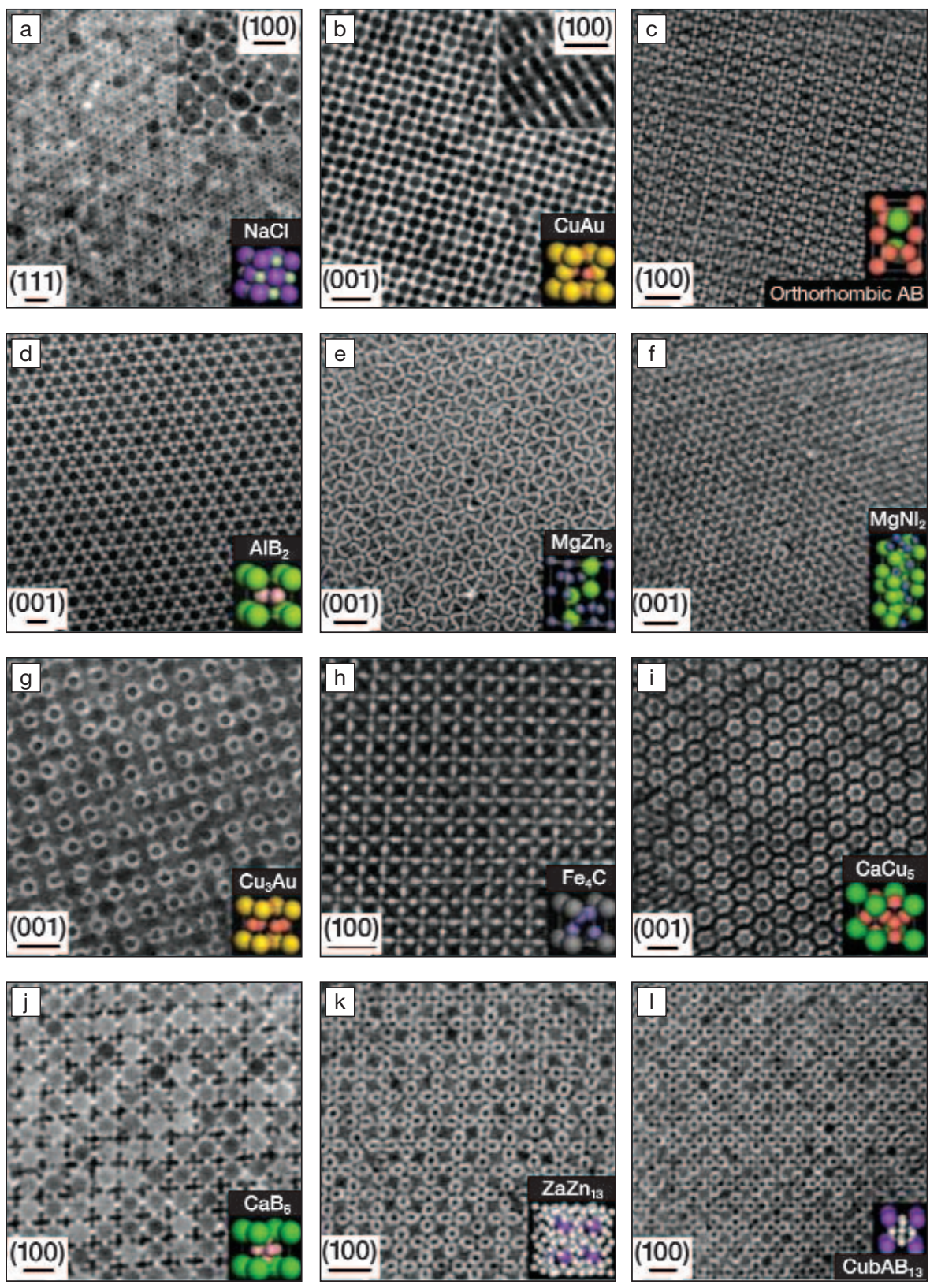

Figure 1. Transmission electron microscope images of some of the latticework structures created by the self-assembly of different nanoparticles. The colored inset image in each frame shows the corresponding three-dimensional structure in an ordinary crystal. Credit: Elena Shevchenko. Published with permission from Nature. () 2006 Nature Publishing Group. 
and $\mathrm{AlB}_{2}$-type superlattices after adding oleic acid....and into $\mathrm{NaZn}_{13}$ - or cuboctahedral $\mathrm{AB}_{13}$-type BNSLs after the addition of dodecylamine or TOPO [tri- $n$-octylphosphine oxide], respectively."

The researchers said that entropic (i.e., space-filling or packing-density factors), as well as van der Waals, steric, and dipolar forces also stabilized the BNSL structures.

\section{Integrated Optical Ring Resonator Demonstrated as a High- Sensitivity Biosensor}

Over the last decade, optical biosensors have become increasingly indispensable tools in the life sciences, particularly in the area of drug research. These sensors need to be simple, reliable, and able to detect and identify extremely low concentrations of biological molecules. In an article in the December 15 issue of Optics Letters (p. 3344), Alex Ksendzov and Ying Lin of the Jet Propulsion Laboratory (JPL) in Pasadena, Calif., report the demonstration of a new optical biosensor based on a ring resonator that is able to detect concentrations of a test protein as low as $0.1 \mathrm{nM}$.

State-of-the-art optical biosensors are based on "whispering gallery" modes, which guide light along the surface of a

\section{Electronic Carriers Cross Si-Bound Alkyl Monolayers in Two Ways}

In order to uncover whether organic molecules can be used to pass electrical current, researchers first need to understand how electrons pass through molecules. "To answer this question," said David Cahen of the Department of Materials and Interfaces at the Weizmann Institute of Science in Israel, "we need to design, build, and use a system that is sufficiently well controlled so that we can be sure that we are measuring what we think we are measuring." Cahen, collaborating with an international group of researchers, has built such a system and has shown that electrons pass through the system by two different mechanisms, switching from one to the other depending on how much voltage is applied and on the length of the molecules.

As reported in the December 31, 2005, issue of Physical Review Letters (DOI: 10.1103/PhysRevLett.95.266807; \#266807) the researchers-Cahen, T. Boecking of the University of New South Wales in Australia, C.K. Chan of Princeton University, and colleagues - studied a system of Si-C linked alkyl monolayers sandwiched between a metal $(\mathrm{Hg})$ and semiconductor $(n-\mathrm{Si})$. They discovered that at low forward-bias voltages, the electrons behave quasi-classically, and the temperaturedependence of the current suggests that current is limited by thermionic emission over a barrier in the semiconductor. At higher forward-bias voltages, the electrons behave as waves, and the dependence of the current on the length of the molecules suggests that current is limited by tunneling through the molecules. The researchers said that the longer the molecules, the lower the voltage at which the molecules start to control the current (see Figure 1).

Control samples of monolayers on silicon were characterized using both photoemission and other spectroscopies as well as advancing water contact angle (CA). Only samples with $\mathrm{CA}>110^{\circ}$ on $\mathrm{Si}$ were used for electrical measurements. With these results and current-voltage measurements, the electron transport processes could be described quantitatively, said the researchers.

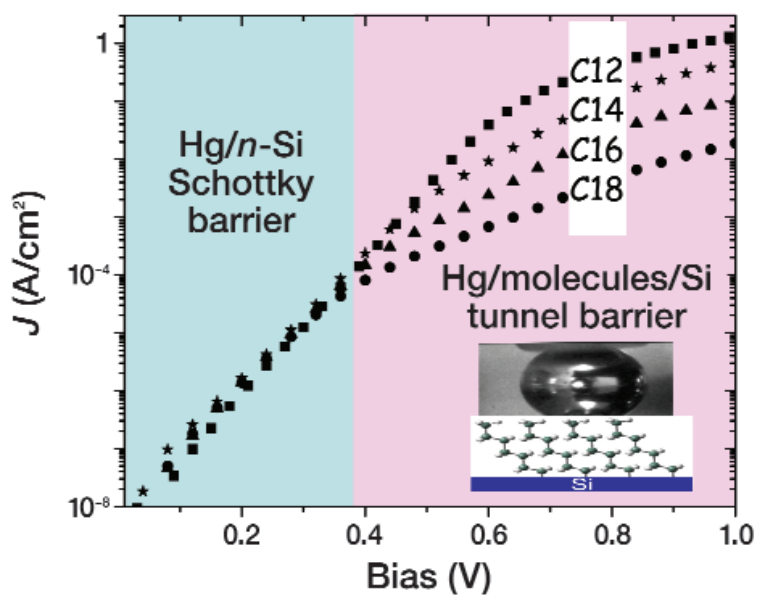

Figure 1. Experimental $\mathrm{n}-\mathrm{Si}-\left(\mathrm{CH}_{2}\right)_{\mathrm{n}-1} \mathrm{CH}_{3} / \mathrm{Hg} \mathrm{J}-\mathrm{V}$ curves.
The researchers said, "Our results agree with theoretical predictions for semiconductor/ insulator / conductor solar cells that had not been seen till now, because no suitable insulator was found."

Cahen added, "Agreement between experiment and theory breaks down, though, for the temperature-dependence of the currentvoltage characteristics, where the signature of the presence of molecules (rather than of, say, an oxide) is seen." spherical microstructure. The term is derived from the Whispering Gallery of St. Paul's Cathedral in London, where the curvature of the dome allows a whisperer near one wall to be heard by a listener near the opposite wall. Proteins binding to the surface of the device alter the index of refraction experienced by the light, and thus change the resonant frequency of the optical mode. However, these modes are numerous and closely spaced in frequency, which complicates the data collection and analysis for small signals.

Instead, the research team's biosensor is based on a 1.8- $\mu$ m-thick $\mathrm{Si}_{x} \mathrm{~N}_{y} / \mathrm{SiO}_{2}$ waveguide bent into a "racetrack" ring resonator with a 13-mm circumference. This device has simple optical modes that are easily identified by transmission resonances. To demonstrate the resonator's sensitivity, the researchers coated its surface with biotin, which has a specific and extremely high affinity for the glycoprotein avidin, and then flowed $3 \mathrm{nM}, 0.6 \mathrm{nM}$, and $0.3 \mathrm{nM}$ concentrations of avidin past the device. By varying the temperature and thus the length of the resonator, the team could locate the transmission resonance frequency. As avidin from the flow accumulated on the resonator surface, the resonance frequency shifted in time, with a rate that was proportional to the protein concentration. Given background drifts, the team estimated that this shift would be measurable for avidin concentrations as low as $0.1 \mathrm{nM}(6.8 \mathrm{ng} / \mathrm{ml})$. This is many orders of magnitude more sensitive than most optical biosensors and may be further enhanced as the team implements plans to improve the quality of the biotin coating and the temperature control and monitoring. Integrated ring resonators carrying a variety of protein-binding coatings may ultimately become the basis for large biosensor arrays for use in pharmaceutical biomolecule identification.

COLIN MCCORMICK

\section{Cellular Uptake of Functionalized Carbon Nanotubes Shown to be Energy-Dependent}

Recent research has shown that singlewalled carbon nanotube (SWNTs) can transport peptides, proteins, and nucleic acids into living cells. The biocompatibility and nontoxicity of SWNTs, together with their near-IR absorptivity, make them a promising new class of biotransporters for drug and radiation therapies. Recent studies conflict on the mechanism that regulates the cellular internalization of SWNTs. One suggests an energy-independent mechanism involving insertion and diffusion of the SWNTs through the cell membrane, while another suggests endocytosis, 